\title{
Static Structure Factor and Static Response Function of Superfluid Helium 4: a Comparative Analysis
}

\author{
Frédéric Caupin · Jordi Boronat • \\ Ken H. Andersen
}

Received: 2 February 2008 / Accepted: 18 March 2008 / Published online: 25 April 2008

(C) Springer Science+Business Media, LLC 2008

\begin{abstract}
Extensive neutron scattering data on liquid helium 4 are available, and have been analyzed to give a number of physical quantities, e.g. static structure factor $S(q)$, excitation energy, and roton linewidth. X-rays also give access to $S(q)$. However, a comprehensive comparison between experimental data and theoretical results, including their dependence on pressure, is still lacking. The static response function $\chi(q)$ has been particularly overlooked, despite its fundamental role in theories of inhomogeneous helium. We present here a critical review about the strength of the main peaks of $S(q)$ and $\chi(q)$. We include in the comparison the analysis of unpublished neutron data and new Monte-Carlo calculations of $\chi(q)$. We find a significant discrepancy between experiments, and suggest corrections which account for some of the differences. We give recommendations for the best values to use for $S(q)$ and $\chi(q)$.
\end{abstract}

Keywords Superfluid helium $4 \cdot$ Static structure factor - Static response function Neutrons · X-rays · Quantum Monte Carlo

PACS 67.25.dt $\cdot$ 61.05.fg $\cdot$ 61.05.cf $\cdot$ 02.70.Ss

F. Caupin $(\varangle)$

Laboratoire de Physique Statistique de 1'Ecole Normale Supérieure, ENS-CNRS-Paris 6-Paris 7, 24 rue Lhomond, 75005 Paris, France

e-mail: caupin@lps.ens.fr

J. Boronat

Departament de Física i Enginyeria Nuclear, Universitat Politècnica de Catalunya, Campus Nord, B4-B5, 08034 Barcelona, Spain

K.H. Andersen

Institut Laue-Langevin, 6 rue Jules Horowitz, B.P. 156, 38042 Grenoble Cedex 9, France 


\section{Introduction}

Superfluid helium 4 has been studied extensively by neutron and X-ray scattering. These techniques give access to valuable information about the microscopic structure of the liquid, such as the static structure factor $S(q)$ and the static response function $\chi(q)$. Surprisingly, a comprehensive comparison between experimental data, and with microscopic calculations, appears to be lacking. This is particularly true when the pressure dependence is considered. In addition, $\chi(q)$ has been somewhat overlooked: there is only one published curve, obtained by Cowley and Woods in 1971 [1] at saturated vapor pressure (svp). This is puzzling when one thinks of the importance of $\chi(q)$ in density functional theory, where the static response can be used as an input $[2,3]$ or a test $[4,5]$ for the functional.

In this paper we present a critical review of the data on $S(q)$ and $\chi(q)$. In Sect. 2 , we recall the methods used to extract these quantities from experiments, and we describe the data selected for our review. Section 3 comprises a brief presentation of the theoretical methods used in our analysis: a sum-rules approach, that allows for estimation of accurate bounds on the magnitudes under discussion, and direct quantum Monte Carlo simulations. Section 4 focuses on the pressure dependence of the peaks of $S(q)$ and $\chi(q)$. We find some inconsistencies between experimental data, and propose a correction. In Sect. 5, we finally make a recommendation for the values to use for the peaks.

\section{Neutron and X-ray Scattering Methods}

A presentation of the scattering techniques used to study liquid helium 4 can be found in [6]. The measurement of the neutron scattering cross section gives access to the dynamic response function $S(q, \omega)$, where $q$ is the momentum transfer, and $\omega$ the energy transfer. At low temperature, $S(q, \omega)$ can be written as

$$
S(q, \omega)=Z(q) \delta[\omega-\omega(q)]+S_{\mathrm{m}}(q, \omega),
$$

where the first term comes from the excitation of a single phonon of energy $\omega(q)$, and the second term includes the contributions from scattering of more than one phonon, the so-called multiphonon part. The observed scattered energy distributions reflect this behavior, with the sharp one-phonon peak broadened by the instrumental resolution, and a broad multiphonon part centered at large $\omega$. The energy moments of $S(q, \omega)$ are of special interest. The $n$-th moment is defined as:

$$
m_{n}=\int_{-\infty}^{+\infty} \omega^{n} S(q, \omega) \mathrm{d} \omega
$$

The zeroth moment is the static structure factor of the liquid $S(q)=m_{0}$ which can also be obtained by X-ray scattering (see below). The static response function $\chi(q)$ is related to the inverse energy weighted moment of $S(q, \omega)$ :

$$
\chi(q)=-2 n \int_{-\infty}^{+\infty} \frac{S(q, \omega)}{\omega} \mathrm{d} \omega=-2 n m_{-1},
$$


Fig. 1 (Color online) The static structure factor (a) and static response function (b) of helium 4 at saturated vapor pressure and $1.1 \mathrm{~K}$, as measured by Cowley and Woods [1] (adapted from their Figs. 12 and 14, respectively). The dotted, dashed, and solid lines show the one phonon, multiphonon, and total contributions, respectively
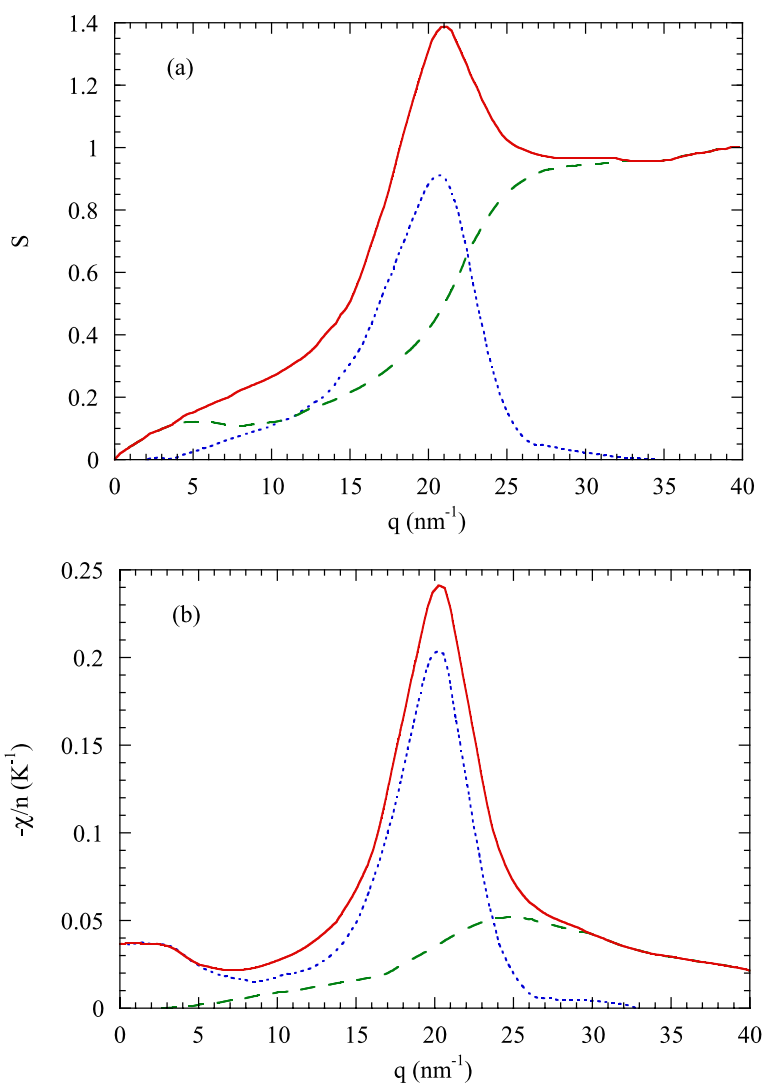

where $n$ is the number of particles per unit volume. The determination of $\chi(q)$ is more accurate than any positive moment because the factor $1 / \omega$ gives more weight to the one-phonon part, and reduces uncertainties due to the high energy tail of the multiphonon part. Figure 1, adapted from the pioneering work of Cowley and Woods [1], allows a comparison of the one phonon and multiphonon contribution to $S(q)$ and $\chi(q) / \mathrm{n}$. It also shows that the one-phonon components are peaked around $q=20 \mathrm{~nm}^{-1}$ : the peak in $S(q)\left(S_{\mathrm{pk}}\right)$ reflects the short range order in the liquid, whereas the peak in $\chi(q) / n\left(\chi_{\mathrm{pk}} / n\right)$ shows that the liquid is more susceptible to perturbations with this spatial period. More specifically, if the liquid is submitted to a periodic external potential

$$
V_{\mathrm{ext}}(\mathbf{r})=V_{\mathbf{q}} \cos (\mathbf{q} \cdot \mathbf{r})
$$

the liquid density shows a modulation around the bulk density $n_{0}$ which can be written to the lowest order as:

$$
n(\mathbf{r})=n_{0}+\chi(q) V_{\mathbf{q}} \cos (\mathbf{q} \cdot \mathbf{r}) .
$$


Table 1 Summary of the experimental data included in our analysis. In the column method, N stands for neutron and X for X-rays. The experimental pressures and lowest temperatures are given. The column data shows the quantities we have taken from the reference to derive the relevant parameters. The column calibration indicates if and how the data were converted to absolute units

\begin{tabular}{|c|c|c|c|c|c|}
\hline Ref. & Method & $\begin{array}{l}P \\
(\mathrm{MPa})\end{array}$ & $\begin{array}{l}\text { Lowest } T \\
\text { (K) }\end{array}$ & Data & Calibration \\
\hline [1] & $\mathrm{N}$ & svp & 1.1 & $S, Z, \chi$ & $\begin{array}{l}\text { Scaled to } S(q)=1 \\
\text { for } q \geq 30 \mathrm{~nm}^{-1}\end{array}$ \\
\hline [7] & $\mathrm{N}$ & $0.1,1,2.4$ & 1.3 & $\omega(q), Z$ & None \\
\hline [8] & $\mathrm{N}$ & $0.07,2.4$ & 1.2 & $S(q, \omega)$ & $\begin{array}{l}\text { Scaled to reproduce } \\
\text { X-ray data for } S(q)\end{array}$ \\
\hline [9] & $\mathrm{N}$ & svp & 1.0 & $S$ & $\begin{array}{l}\text { Scaled to } S(q)=1 \\
\text { at large } q\end{array}$ \\
\hline [10] & $\mathrm{N}$ & $\begin{array}{l}0,0.2,0.5 \\
1,1.5,2\end{array}$ & 0.5 & $\begin{array}{l}\omega(q), Z, \\
S(q, \omega)\end{array}$ & $\begin{array}{l}\text { Scaled to reproduce at svp } \\
S\left(q=15.1 \mathrm{~nm}^{-1}\right)=0.5673\end{array}$ \\
\hline [11] & $\mathrm{N}$ & $0.1,1.1,2.4$ & 0.6 & $S(q, \omega)$ & None \\
\hline [12] & $\mathrm{X}$ & svp & 0.79 & $S$ & Neon gas \\
\hline [13] & $\mathrm{X}$ & $0,0.5,1.1,1.7,2.2$ & 1.67 & $S$ & Neon gas \\
\hline [14] & $\mathrm{X}$ & $0.3,1.3,2.3$ & 1.16 & $S$ & Neon gas \\
\hline
\end{tabular}

In order to carry out a comparison between different data we have focused our study on the strength of the main peak. In this way, we can simplify the analysis and be quantitatively accurate.

There are many references with neutron scattering data on superfluid helium 4 available. We have selected here those which give information about $S(q), Z(q)$, and/or $\chi(q)$ and their pressure dependence, including neutron [1, 7-11] and X-ray [12-14] scattering data. Table 1 summarizes the experimental conditions; when absolute units are used, the corresponding calibration method is described. The work of Cowley and Woods [1] is the only one showing $\chi(q)$ explicitly (see Fig. 1b). Cowley and Woods [1] and Svensson et al. [9] give directly $S(q)$. Some other papers give $S(q, \omega)$ in absolute units, allowing a calculation of the zeroth moment $S(q)$ and of $\chi(q)$ through (3); note that this gives more or less accurate lower bounds depending on the maximum energy transfer produced in the experiments. When the amplitude of the one-phonon part $Z(q)$ is given, we use it directly to estimate the one-phonon contribution to $\chi(q) / n$ as $Z(q) / \omega(q)$, according to (1) and (3). Finally, the other papers included in our analysis are those which do not provide an absolute scale, but give measurements at several pressures; after normalization to a reference pressure, their pressure dependence can be compared to that of absolute measurements (see Sect. 4). Reference [11] corresponds to unpublished data from the experimental runs described in [15].

X-rays have the suitable wavelengths $\left(\simeq 10 \mathrm{~nm}^{-1}\right)$ to be diffracted by liquids, but their energy $\left(\simeq 10^{4} \mathrm{eV}\right)$ is much higher than that of thermal neutrons $\left(\simeq 10^{-2} \mathrm{eV}\right)$. The relevant energy transfers being around $\simeq 10^{-3} \mathrm{eV}, \mathrm{X}$-rays integrate over all energies, leading directly to $S(q)$. Among the early X-ray data, the first accurate work in the peak region is from Achter and Meyer [12]. We have also used the more re- 
Fig. 2 (Color online) The peak of the one phonon contribution $Z_{\mathrm{pk}}$ to the static response function of helium 4 as a function of temperature for three pressures. The data are taken without correction from Gibbs et al. [10] (see Sect. 4.1 for details)

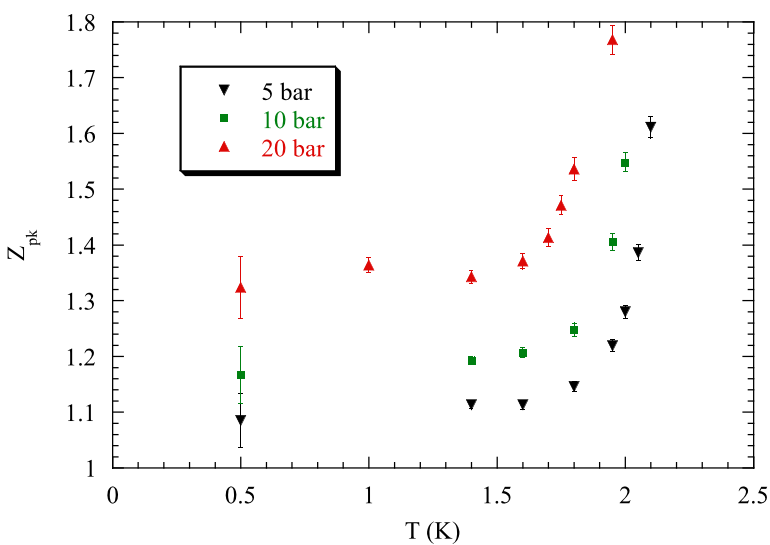

cent data of [14]. On the other hand, we have discarded earlier data from the same group [16, 17] because an error in the calibration was pointed out [14]; yet we have included the corrected values from their earlier data set [16, 17] as given by this group [13]. This calibration error has been overlooked in a review about liquid helium properties at svp [18], where only [9] and [16] were considered, leading to a discrepancy in the region of the peak, and to the arbitrary choice of a spline near the average of the two sets of data as the recommended value for $S(q)$. The experimental conditions of the X-ray experiments we have selected are given in Table 1.

All data were copied from the original tables or extracted from the published figures; when both table and figure were available, the table was preferred. When several temperatures had been investigated, only the data at the lowest one were kept for the comparison.

As the experimental data compared in this review were taken at different temperatures (see Table 1), we need to consider the temperature dependence of the parameters. The X-ray data of Wirth and Hallock [14] show that $S(q)$ increases by around $5 \%$ when the temperature increases up to $T_{\lambda}$; below $1.3 \mathrm{~K}, S(q)$ can be taken as constant, within the experimental uncertainties. The temperature variation of $Z(q)$, extracted from the neutron data, depends on the method used for the decomposition between the one and multi-phonon components. However, the methods give identical results below 1.3 K [19,20]. More recent data [10] (using the simple subtraction method) go down to $0.5 \mathrm{~K}$, and show that $Z(q)$ can be taken as constant below $1.5 \mathrm{~K}$ (see Fig. 2). $S(q)$ was measured at $1.67 \mathrm{~K}$ in [13], which leads to slightly higher values than at low temperature, especially for high pressures. All the other data we have selected, although taken at different temperatures, can be directly compared.

\section{Theoretical Approaches}

Microscopic theories have been extensively applied to the study of liquid helium 4 and, with the help of available accurate interatomic He-He potentials, have reproduced a lot of experimental data both at zero and finite temperature. In the present work, we focus our selection of theoretical data on the three functions $S(q), Z(q)$, 
and $\chi(q)$ to the bounds obtained by using a sum-rules approach and to results obtained at zero temperature by direct quantum Monte Carlo (QMC) simulations.

\subsection{Sum-Rules Approach}

The lowest order sum rules have been extensively used in the study of quantum liquids. Using proper combinations of them one can calculate approximate values of relevant properties of the system such as the excitation spectrum or the static response. Obviously, their calculation is easier than explicitly excited-states calculations since only ground-state properties of the liquid are required. These ground-state properties are nowadays available with high accuracy from QMC simulations. This analysis was carried out by Boronat et al. [21] and we review here their main arguments.

The ratio $m_{1} / m_{0}$ is the well-know upper bound on $\omega(q)[22,23]$ :

$$
\omega(q) \leq \frac{\hbar q^{2}}{2 M S(q)},
$$

where $M$ is the particle mass. One may think of checking the consistency of the data on $S(q)$ by using (6) and the data on the dispersion curve $\omega(q)$. Unfortunately, the multiphonon contribution is so large in the peak region of $S(q)$ that the upper bound in (6) overestimates $\omega(q)$ by too much to be of practical use.

There are also theoretical constraints on $\chi(q) / n$. The limits at small and large wavenumbers are fixed: $1 /\left(M c^{2}\right)$ (compressibility sum rule) and $4 M /\left(\hbar^{2} q^{2}\right)$ (freeparticle limit), respectively, where $c$ is the speed of sound. Lower and upper bounds can also be given on $m_{-1}$. A first approach, using the Feynman approximation [24], leads to:

$$
\frac{2 M m_{0}^{2}}{\hbar^{2} q^{2}} \leq \frac{1}{n} \chi(q) \leq \frac{m_{0}}{\hbar \omega(q)} .
$$

These two bounds would collapse if the excitation spectrum was reduced to the single phonon-roton mode. Because of multiphonon scattering, the bounds are relatively different, and may just serve as an internal consistency test that should be passed by all neutron data.

Better bounds were later derived by Dalfovo and Stringari [25]:

$$
\begin{aligned}
\frac{2 M m_{0}^{2}}{\hbar^{2} q^{2}} \frac{1}{1-\frac{\Delta(q)}{\epsilon(q)}} & \leq \frac{1}{n} \chi(q) \\
& \leq \frac{m_{0}}{\hbar \omega(q)}\left[1-\frac{m_{0}}{m_{1}}\left(\frac{m_{1}}{m_{0}}-\hbar \omega(q)\right)^{2}\left(\frac{m_{2}}{m_{1}}-\hbar \omega(q)\right)^{-1}\right],
\end{aligned}
$$

with

$$
\epsilon(q)=\left[\frac{m_{3}}{m_{1}}+\left(\frac{m_{1}}{m_{0}}\right)^{2}-2 \frac{m_{2}}{m_{0}}\right]\left[\frac{m_{2}}{m_{1}}-\frac{m_{1}}{m_{0}}\right]^{-1}
$$

and

$$
\Delta(q)=\frac{m_{2}}{m_{1}}-\frac{m_{1}}{m_{0}}
$$


As the high-order moments are less accurately known experimentally because of the increasing multiphonon contribution, these bounds can only be calculated theoretically. In particular, the fact that positive moments can be expressed in terms of ground-state properties allows their calculation by standard QMC techniques. The above equation thus gives boundaries for $\chi(q) / n$ [21], whose direct calculation requires a more accurate QMC scheme.

\subsection{Direct Calculations}

The diffusion Monte Carlo (DMC) method is nowadays the most accurate microscopic approach for studying quantum fluids at zero temperature. DMC is devised to solve the imaginary-time Schrödinger equation in a stochastic way. When the interacting particles of the fluid obey Bose statistics DMC provides exact results, within some statistical uncertainties. For further technical details on the DMC method see [26].

The static structure function $S(q)$ is a ground-state function which can be accurately calculated using its definition,

$$
S(q)=\frac{1}{N} \frac{\left\langle\Phi_{0}\left|\rho_{-\mathbf{q}} \rho_{\mathbf{q}}\right| \Phi_{0}\right\rangle}{\left\langle\Phi_{0} \mid \Phi_{0}\right\rangle},
$$

with $\rho_{\mathbf{q}}=\sum_{i=1}^{N} e^{i \mathbf{q} \cdot \mathbf{r}_{i}}$ the fluctuation-density operator and $N$ the number of particles $[27,28]$.

The strength $Z(q)$ of the single excitation peak of $S(q, \omega)$ can also be calculated using DMC [29]. In this case, the results are not exact but they are very accurate since the excited wave function incorporates Feynman-Cohen backflow correlations. More specifically, $Z(q)$ is obtained by computing the following expectation value

$$
Z(q)=\frac{\left|\left\langle\rho_{-\mathbf{q}} \rho_{\mathbf{q}}^{\mathrm{BF}}\right\rangle_{0}\right|^{2}}{\left\langle\rho_{-\mathbf{q}}^{\mathrm{BF}} \rho_{\mathbf{q}}^{\mathrm{BF}}\right\rangle_{0}},
$$

sampled in the ground-state configurations generated by the DMC method. The backflow operator is given by

$$
\rho_{\mathbf{q}}^{\mathrm{BF}}=\sum_{i=1}^{N} e^{i \mathbf{q} \cdot \tilde{\mathbf{r}}_{i}},
$$

with

$$
\tilde{\mathbf{r}}_{i}=\mathbf{r}_{i}+\sum_{j \neq i} \eta\left(r_{i j}\right) \mathbf{r}_{i j}
$$

As usual, the function $\eta(r)$ entering in the backflow wave function (14) has been chosen to be a Gaussian $\eta(r)=A \exp \left[-\left(\left(r-r_{b}\right) / \omega_{b}\right)^{2}\right]$, with $A, r_{b}$, and $\omega_{b}$ parameters to be optimized.

We also mention an alternative used to study ground and excited states of helium: the variational Monte Carlo method with shadow wave functions (SWF). Moroni et al. have used SWF to calculate $S(q)$ and $Z(q)$ at svp and freezing pressures [28]. 
The DMC calculation of the static response $\chi(q)$ requires a more specific implementation. We have followed the method used by Moroni et al. [30], extending the calculations to more densities to better study the pressure dependence of $\chi(q)$. The simulation is carried out introducing in the Hamiltonian of the system an external potential inducing a modulation of the density,

$$
V_{\mathrm{ext}}(\mathbf{R})=\sum_{i=1}^{N} V_{q} \cos \left(\mathbf{q} \cdot \mathbf{r}_{i}\right) .
$$

In this model potential, $\mathbf{q}=2 \pi / L\left(n_{x}, n_{y}, n_{z}\right)$ is a momentum vector compatible with the periodic boundary conditions for a cubic box of size $L$ and $V_{q}$ modulates the strength of the interaction. The energy per particle of the system can be expanded in even powers of the strength $V_{q}$,

$$
(E / N)\left(V_{q}\right)=(E / N)_{0}+C_{2} V_{q}^{2}+C_{4} V_{q}^{4}+\ldots,
$$

for any given momentum $\mathbf{q},(E / N)_{0}$ being the ground state energy $\left(V_{q}=0\right)$. The second-order coefficient in the expansion (16) gives the static response: $\chi(q) / n=$ $4 C_{2}$. For each momentum $q$ one carries out simulations with increasing values of $V_{q}$ and extracts the static response from a fit (16) to the DMC energies.

\section{Comparative Analysis of Results}

We have focused our comparative analysis between the available experimental data and theoretical results on the strength of the main peak. In this way, the comparison is simplified and an accurate quantitative analysis can be carried out.

\subsection{Discrepancies and Proposed Corrections}

As we shall see in the next section, the data we have gathered are in relatively good agreement with each other. However, two sets of data [7, 10] exhibited a marked discrepancy, especially obvious in $Z_{\mathrm{pk}}$ and $\chi_{\mathrm{pk}} / n$. We have taken the recent data from Gibbs et al. [10] using the following procedure. The one phonon contribution $Z_{\mathrm{pk}}$ was taken from the tables in Appendix A of Gibbs's thesis. These data agree well with others at svp, but they exhibit too large a pressure dependence, leading to a value approximately $30 \%$ higher at $2 \mathrm{MPa}$. This too large pressure dependence compared to other data is also found in $\chi_{\mathrm{pk}} / n$. In addition, the data at $2 \mathrm{MPa}$ even fall just above the theoretical upper bound (see Sect. 3.1), but it is still compatible with the error bars. However, if we include the multiphonon contribution to calculate $\chi_{\mathrm{pk}} / n$, the data at $2 \mathrm{MPa}$ clearly falls above the theoretical upper bound. The multiphonon contribution to $S_{\mathrm{pk}}$ was estimated by extracting the data for $S(q, \omega)$ at $q=20 \mathrm{~nm}^{-1}$ from Fig. 19 of Gibbs et al. [10], and integrating them from $\omega=12 \mathrm{~K}$ (to avoid the tail of the one phonon peak convoluted with the experimental resolution) to $40 \mathrm{~K}$; we expect this to be an underestimate, because the multiphonon peak extends to higher energies. 
Fig. 3 (Color online) The peak of the static structure factor $S_{\mathrm{pk}}$ of helium 4 as a function of pressure. In addition to neutron data [1, 8-10], we have included X-ray measurements [12-14] and QMC calculations [27, 28].

The correspondence between symbols and references is given in the legend. The empty symbols correspond to data in absolute units, whereas the filled symbols [8] correspond to data rescaled to fit previous X-ray data. No error bars were given in [8] and [28]. The data from [10] have been corrected for density variation (see Sect. 4.1)

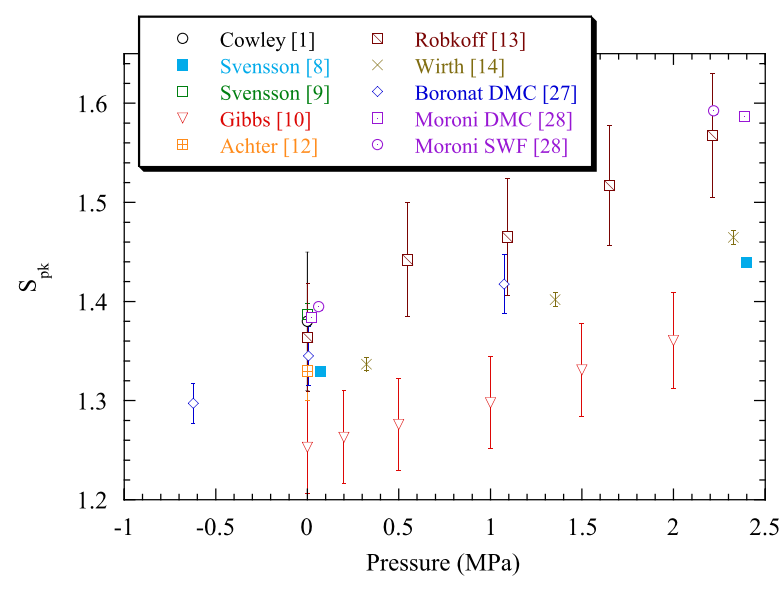

This finding leads us to reconsider the calibration of Gibbs's data. Looking at Gibbs' thesis, we have found that the data for $S(q)$ were rescaled at svp by a constant factor to fit a previous value at $q=15.1 \mathrm{~nm}^{-1}, 0.5673$ [10]. This value appears to be taken from the corrected X-ray data of Robkoff and Hallock at svp [13]. However, the same scaling factor seems to have been used at all other pressures (see Gibbs' thesis [10]). This introduces a systematic error, because the neutron scattering signal is proportional to the number of scattering atoms, which will increase with pressure simply because of the increase of the liquid density. The data should therefore be further divided by a factor $\rho(P) / \rho(P=0)$, which varies by about $19 \%$ between svp and freezing. Using for $\rho(P)$ the formula given in the Appendix, we obtain a better agreement.

Figure 11 of [7] gives $Z(q)$ on an arbitrary scale, at $0.1,1$ and $2.4 \mathrm{MPa}$. If we extrapolate the value of $Z_{\mathrm{pk}}$ to zero pressure, and rescale it to fit previous data [1], we can extract the pressure dependence of $Z_{\mathrm{pk}}$. It is very close to the uncorrected data from Gibbs et al. [10], and there is no indication in [7] that the intensities have been corrected for the pressure-dependence of the density. Consequently, we have applied the same density correction to the data of [7]. We have used the same procedure to extract the pressure dependence of $\chi_{\mathrm{pk}}$.

\subsection{Summary of the Pressure Dependencies}

We have chosen to plot the different peak values as a function of pressure. Most often, the experimental pressure is given in the original work. However, in [13, 14], the density is given instead. The DMC, SWF, and DFT calculations use the density as input. The pressure is a derived quantity: although the different calculations give pressures in good agreement with each other and with experiments in the stable liquid region [3, 27], they start to deviate in the metastable region at high pressure (see [31] and the Appendix). For consistency, we chose to convert all the densities into pressure using an equation of state based on experimental data, as described in the Appendix.

Figure 3 shows a comparison between data for the pressure dependence of $S_{\mathrm{pk}}$. $\mathrm{X}$-ray data are found to be consistent with QMC calculations. Recalculated data from 


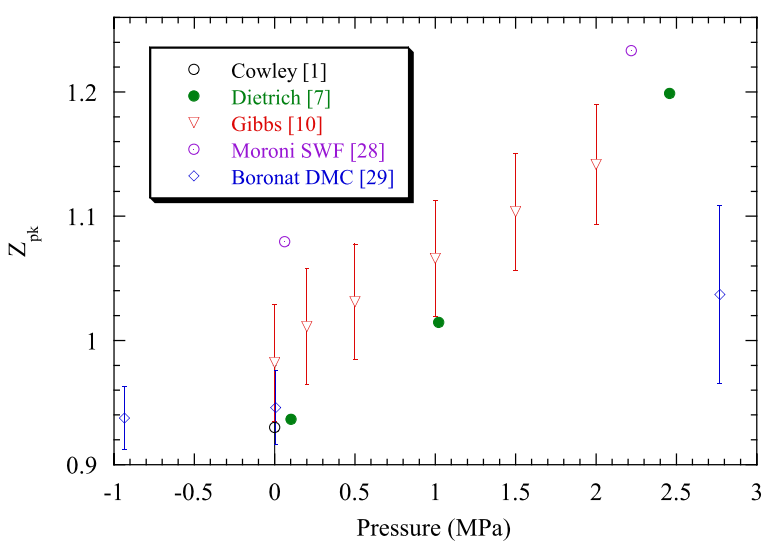

Fig. 4 (Color online) The peak of the one phonon contribution $Z_{\mathrm{pk}}$ to the static response function of helium 4 as a function of pressure. In addition to neutron data $[1,7,10]$, we have included QMC calculations [28, 29]. The correspondence between symbols and references is given in the legend. The empty symbols corresponds to data in absolute units, whereas the filled symbols [7] correspond to data that we have rescaled so that $Z_{\mathrm{pk}}$ extrapolates at svp to the value of Cowley and Woods [1] in order to show their pressure variation. No error bars were given in [7] and [28]. The data from [7] and [10] have been corrected for density variation (see Sect. 4.1)

Robkoff and Hallock [13] are systematically higher than the ones from Wirth and Hallock [14]: this can be ascribed in part to the higher temperature investigated in [13] (see Sect. 2); we note however that Wirth and Hallock [14] also studied the same temperatures and densities as in [13] for comparison, and still found a few percents discrepancy. Early neutron data at svp [1,9] give slightly too large a peak. This is quite surprising because the scattered intensity was scaled in order to get $S(q)=1$ for large $q$. We would expect this procedure to give reliable results. However, we note that the error bars of [1] are large enough to be still compatible with X-ray data. This is not the case for the data from [9]: one possibility is that the quoted $0.8 \%$ statistical precision and less than $1 \%$ systematic error are underestimated. The early data from Svensson et al. [8] agree well with X-ray data at svp because it was rescaled for this purpose. In addition, we note that it also gives a consistent pressure dependence. After the correction described in Sect. 4.1, the data from [10] also show a consistent pressure dependence, although the absolute value is somewhat lower than others; this is likely due to an underestimate of the multiphonon contribution, because of the limited energy range investigated $(\omega \leq 4 \mathrm{meV}[10])$.

Figure 4 shows a comparison between neutron data for the pressure dependence of $Z_{\mathrm{pk}}$. The data with absolute calibration $[1,10]$ agree at svp. When needed, the other data have been rescaled so that they extrapolate at svp to the results of [1]. This allows a comparison of the pressure dependence. The data from [7] and [10] agree with each other. After the correction described in Sect. 4.1, they fall in between the two theoretical MC calculations of $Z_{\mathrm{pk}}[28,29]$. These calculations differ by around 10$20 \%$, a larger difference than the one observed for $S_{\mathrm{pk}}$ (see Fig. 3). This fact can be understood taking into account that the calculation of $S(q)$ is exact within statistical accuracy, whereas the one of $Z(q)$ is variational (see Sect. 3.2). As calculations of 


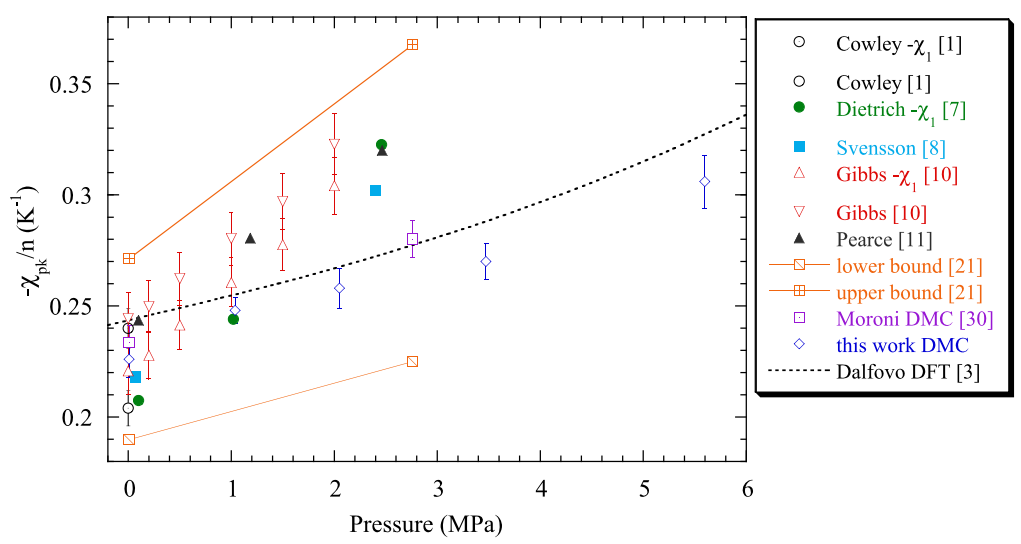

Fig. 5 (Color online) The peak of the static response function $\chi_{\mathrm{pk}}$ of helium 4 as a function of pressure. The correspondence between symbols and references is given in the legend. The empty symbols correspond to data in absolute units, whereas the filled symbols correspond to data rescaled either in the original work to fit previous data [8], or by us $[7,11]$ so that $\chi_{\mathrm{pk}}$ extrapolates at svp to the value of Cowley and Woods [1] in order to show their pressure variation. The data from [7] and [10] have been corrected for density variation (see Sect. 4.1). The theoretical bounds on $\chi_{\mathrm{pk}}$ (see Sect. 3.1 and [21]) are connected with straight lines to guide the eye. The symbol $\chi_{1}$ in the legend indicates that only the one-phonon part of $\chi_{\mathrm{pk}}$ was taken into account. Direct QMC calculations of $\chi_{\mathrm{pk}}$ are also shown. The dotted line gives the DFT value of $\chi_{\mathrm{pk}}$, which was adjusted on the value of Cowley and Woods [1], and included a pressure variation close to the one of [30]

$Z(q)$ reported in Fig. 4 rely on two different models for the excited wave function, the results differ more significantly.

Figure 5 shows a comparison between data for the pressure dependence of $\chi_{\mathrm{pk}} / n$. We have included the theoretical values: bounds from the sum-rules approach (Sect. 3.1) [21], QMC calculations ([30] and this work, see Sect. 3.2), and the data from Orsay-Trento (OT) density functional theory (DFT) [3], which was adjusted to reproduce the data of Cowley and Woods [1], and includes a pressure dependence that follows the results of the QMC calculations [30]. The absolute values [1, 10] agree well at svp. The same procedure as above has been used for data given in relative units: we have rescaled the data from [7] and [11] so that their extrapolation at svp coincides with the one-phonon and total contribution to $\chi_{\mathrm{pk}} / n$ in [1], respectively. After the correction described in Sect. 4.1, the data of [7] and [10] fall between the theoretical bounds. We find a reasonable agreement between all neutron data. The experimental values of $\chi_{\mathrm{pk}} / n$ at high pressure are somewhat higher than the theoretical predictions, which are consistent with each other; it would be interesting to repeat the measurements near the freezing pressure to check this issue.

\section{Conclusion}

The detailed review of data on the static structure factor $S$, its one-phonon contribution $Z$, and the static response function $\chi$ of superfluid helium 4 leads to an overall good agreement. In the course of the comparison, two sets of data [7, 10] were found 
Table 2 Experimental data from Gibbs et al. [10] after the correction described in Sect. 4.1. The error bars are given in parenthesis. The value of $S_{\mathrm{pk}}$ is given for completeness, although it is obviously too low (see Fig. 3), presumably because of the limited energy range investigated $(\omega \leq 4 \mathrm{meV}[10]) . Z_{\mathrm{pk}}$ and $\chi_{\mathrm{pk}}$ are not affected by this limitation, and we recommend their use.

\begin{tabular}{llll}
\hline Pressure (MPa) & $S_{\mathrm{pk}}$ & $Z_{\mathrm{pk}}$ & $-\chi_{\mathrm{pk}} / n\left(\mathrm{~K}^{-1}\right)$ \\
\hline 0 & $1.253(0.047)$ & $0.982(0.047)$ & $0.244(0.012)$ \\
0.2 & $1.263(0.047)$ & $1.011(0.047)$ & $0.250(0.012)$ \\
0.5 & $1.276(0.047)$ & $1.031(0.047)$ & $0.262(0.012)$ \\
1 & $1.298(0.047)$ & $1.066(0.047)$ & $0.280(0.012)$ \\
1.5 & $1.331(0.047)$ & $1.104(0.047)$ & $0.297(0.013)$ \\
2 & $1.361(0.048)$ & $1.142(0.048)$ & $0.323(0.014)$ \\
\hline
\end{tabular}

to give too high values. The most likely explanation is the absence of a density factor required to account for the change in the number of scattering atoms, and we found that including this factor removes most of the discrepancy. We have also reviewed the theoretical results for $S_{\mathrm{pk}}, Z_{\mathrm{pk}}$, and $\chi_{\mathrm{pk}}$, and provided new ones for $\chi_{\mathrm{pk}}$. There is an overall agreement for $S_{\mathrm{pk}}$ and $\chi_{\mathrm{pk}}$ between the results of two groups ([27], [29] and this work on one hand, and [28] and [30] on the other), the difference being only statistically significant for $Z_{\mathrm{pk}}$ (see Fig. 4), due to the variational character of its estimation and the use of different models for the excited wave function. Of more concern is the fact that both theoretical results fall below the experimental values for $\chi_{\mathrm{pk}}$ near freezing; it would be interesting to perform new measurements to clarify this issue.

Because $S$ is sensitive to the multiphonon scattering at large energy transfer, X-ray data are regarded as more reliable for this quantity. The most accurate are those of Wirth and Hallock [14]. However, they were unfortunately not taken at svp, which often serves as a reference. We note also that the corrected data from Robkoff and Hallock [13], which included svp, but were performed at higher temperature, fall slightly above the data from Wirth and Hallock [14].

The multiphonon scattering is less important for $Z$ (by definition) and for $\chi$ (because of the weight $1 / \omega$ in (3)). In addition, these quantities are not available with $\mathrm{X}$-rays. Consequently, we recommend to use the corrected neutron data from Gibbs $e t$ $a l$. [10], which give the most detailed pressure dependence. The values of $Z_{\mathrm{pk}}$ and $\chi_{\mathrm{pk}}$ are given in Table 2. However, we note that these values are higher than the theoretical predictions at high pressure (see Fig. 5), and that we have introduced a density factor for the correction (see Sect. 4.1). Although the agreement with other data makes us confident with this correction, new measurements would be welcome, including also a pressure closer to freezing.

The precise value of $\chi_{\mathrm{pk}}$ has a strong impact on DFT of freezing [32-35]. Using the formula from the OT DFT, the solid was found to be always more stable than the liquid; a reduction by $7 \%$ allowed to recover the experimental freezing pressure $[34,35]$. Unfortunately, this goes in the wrong direction, as the experimental data point to a value of $\chi_{\mathrm{pk}}$ higher than the OT one (see Fig. 5). By comparing with a more accurate calculation [36], it was also shown that the second order truncation used in the simple DFT of freezing is problematic [37]. Anyhow, as the peak of $\chi$ 
gives the susceptibility of the liquid to a perturbation at a wavelength corresponding to the crystal lattice parameter, its knowledge near freezing should be incorporated in the theoretical descriptions of freezing.

Acknowledgements F.C. acknowledges support from ANR grant 05-BLAN-0084-01, and J.B. from DGI (Spain) Grant No. FIS2005-04181 and Generalitat de Catalunya Grant No. 2005SGR-00779. We thank W. Montfrooij, J.V. Pearce and W.G. Stirling for helpful discussions, and for communicating their data.

\section{Appendix: Equation of State}

To convert density into pressure, we have chosen to use an equation of state based on the experimental data of [38], which provides accurate values for the molar volume of liquid helium 4 at $50 \mathrm{mK}$ between 0 and $2.45 \mathrm{MPa}$. The original work used a 9th order polynomial interpolation to represent the data. Unfortunately, the corresponding $P(\rho)$ shows a large, unphysical increase in slope when used to extrapolate the data in the negative pressure range. Consequently, we have chosen to fit the original data with a simpler formula, originally proposed by Maris [39]. We convert the molar volume into density using the molar mass of helium 4, $m_{4}=4.0026032 \times 10^{-3} \mathrm{~kg} \mathrm{~mol}^{-1}$ [38], and fit the data with:

$$
P=P_{\mathrm{s}}+\frac{b^{2}}{27}\left(\rho-\rho_{\mathrm{s}}\right)^{3} .
$$

The parameters are: $P_{\mathrm{s}}=-0.994921 \mathrm{MPa}, b=0.0137222 \mathrm{~m}^{3} \mathrm{~s}^{-1} \mathrm{~kg}^{-1}$, and $\rho_{\mathrm{s}}=$ $92.9036 \mathrm{~kg} \mathrm{~m}^{-3} . P_{\mathrm{s}}$ and $\rho_{\mathrm{s}}$ are the coordinates of the liquid-vapor spinodal point, where the liquid phase becomes unstable [39]. Equation (17) reproduces the experimental data within $1.5 \mathrm{kPa}$, and provides a smooth extrapolation in the metastable regions. When compared to the EOS from DFT or DMC calculations, the agreement is good below the freezing pressure, but the difference increases with increasing pressure: at $\rho=193 \mathrm{~kg} \mathrm{~m}^{-3}$, the present EOS gives $P=6 \mathrm{MPa}, 0.1 \mathrm{MPa}$ above the pressure from the OT DFT [3], and $1 \mathrm{MPa}$ below that of DMC [31].

\section{References}

1. R.A. Cowley, A.D.B. Woods, Can. J. Phys. 49, 177 (1971)

2. C. Ebner, W.F. Saam, Phys. Rev. B 12, 923 (1975)

3. F. Dalfovo, A. Lastri, L. Pricaupenko, S. Stringari, J. Treiner, Phys. Rev. B 52, 1193 (1995)

4. J. Dupont-Roc, M. Himbert, N. Pavloff, J. Treiner, J. Low Temp. Phys. 81, 31 (1990)

5. L. Szybisz, I. Urrutia, Phys. Lett. A 338, 155 (2005)

6. A.D.B. Woods, R.A. Cowley, Rep. Prog. Phys. 36, 1135 (1973)

7. O.W. Dietrich, E.H. Graf, C.H. Huang, L. Passell, Phys. Rev. A 5, 1377 (1972)

8. E.C. Svensson, P. Martel, V.F. Sears, A.D.B. Woods, Can. J. Phys. 54, 2178 (1976)

9. E.C. Svensson, V.F. Sears, A.D.B. Woods, P. Martel, Phys. Rev. B 21, 3638 (1980)

10. M.R. Gibbs, K.H. Andersen, W.G. Stirling, H. Schober, J. Phys. Condens. Matter 11, 603 (1999). M.R. Gibbs, PhD thesis, Keele University (1996)

11. J.V. Pearce, Unpublished data

12. F.K. Achter, L. Meyer, Phys. Rev. 188, 291 (1969) 
13. See AIP document No. PAPS PRBMD-35-116-37 which provides recalculated values of the data from $[17,18]$

14. F.H. Wirth, R.B. Hallock, Phys. Rev. B 35, 89 (1987)

15. J.V. Pearce, J. Bossy, H. Schober, H.R. Glyde, D.R. Daughton, N. Mulders, Phys. Rev. Lett. 93, 145303 (2001)

16. H.N. Robkoff, R.B. Hallock, Phys. Rev. B 24, 159 (1981)

17. H.N. Robkoff, R.B. Hallock, Phys. Rev. B 25, 1572 (1982)

18. R.J. Donnelly, C.F. Barenghi, J. Phys. Chem. Ref. Data 27, 1217 (1998)

19. E.F. Talbot, H.R. Glyde, W.G. Stirling, E.C. Svensson, Phys. Rev. B 38, 11229 (1988)

20. K.H. Andersen, W.G. Stirling, J. Phys. Condens. Matter 6, 5805 (1994)

21. J. Boronat, J. Casulleras, F. Dalfovo, S. Stringari, S. Moroni, Phys. Rev. B 52, 1236 (1995)

22. A. Bijl, Physica 7, 869 (1940)

23. R.P. Feynman, Phys. Rev. 94, 262 (1954)

24. D. Hall, E. Feenberg, Ann. Phys. 63, 335 (1971)

25. F. Dalfovo, S. Stringari, Phys. Rev. B 46, 13991 (1992)

26. B.L. Hammond, W.A. Lester Jr., P.J. Reynolds, Monte Carlo Methods in Ab Initio Quantum Chemistry (World Scientific, Singapore, 1994)

27. J. Boronat, J. Casulleras, Phys. Rev. B 49, 8920 (1994)

28. S. Moroni, D.E. Galli, S. Fantoni, L. Reatto, Phys. Rev. B 58, 909 (1998)

29. J. Boronat, J. Casulleras, J. Low Temp. Phys. 110, 443 (1998)

30. S. Moroni, D.M. Ceperley, G. Senatore, Phys. Rev. Lett. 69, 1837 (1992)

31. L. Vranješ, J. Boronat, J. Casulleras, C. Cazorla, Phys. Rev. Lett. 95, 145302 (2005)

32. F. Dalfovo, J. Dupont-Roc, N. Pavloff, S. Stringari, J. Treiner, Europhys. Lett. 16, 205 (1991)

33. S. Moroni, G. Senatore, Europhys. Lett. 16, 373 (1991)

34. F. Caupin, T. Minoguchi, J. Low Temp. Phys. 134, 181 (2004)

35. F. Caupin, T. Minoguchi, J. Low Temp. Phys. 138, 331 (2004)

36. F. Ancilotto, M. Barranco, F. Caupin, R. Mayol, M. Pi, Phys. Rev. B 72, 214522 (2005)

37. F. Caupin, F. Ancilotto, M. Barranco, R. Mayol, M. Pi, J. Low Temp. Phys. 148, 731 (2007)

38. E. Tanaka, K. Hatakeyama, S. Noma, T. Satoh, Cryogenics 40, 365 (2000)

39. H.J. Maris, Phys. Rev. Lett. 66, 45 (1991) 